\title{
Mathematical Transform of Traveling-Wave Equations and Phase Aspects of Quantum Interaction
}

\author{
Ezzat G. Bakhoum ${ }^{\mathbf{1}}$ and Cristian Toma ${ }^{2}$ \\ ${ }^{1}$ Department of Electrical and Computer Engineering, University of West Florida, \\ 11000 University Parkway, Pensacola, FL. 32514, USA \\ ${ }^{2}$ Faculty of Applied Sciences, Politechnica University, Hagi-Ghita 81, 060032 Bucharest, Romania \\ Correspondence should be addressed to Ezzat G. Bakhoum, ebakhoum@uwf.edu
}

Received 28 July 2009; Accepted 30 August 2009

Academic Editor: Carlo Cattani

Copyright (C) 2010 E. G. Bakhoum and C. Toma. This is an open access article distributed under the Creative Commons Attribution License, which permits unrestricted use, distribution, and reproduction in any medium, provided the original work is properly cited.

The traveling wave equation is an essential tool in the study of vibrations and oscillating systems. This paper introduces an important extension to the Fourier/Laplace transform that is needed for the analysis of signals that are represented by traveling wave equations. Another objective of the paper is to present a mathematical technique for the simulation of the behavior of large systems of optical oscillators.

\section{Introduction}

The Fourier and the Laplace transforms are great mathematical tools that have served science well for nearly two centuries. Recently, however, the Fourier/Laplace transform has become increasingly inadequate for treating one specific class of signals, namely, signals represented by traveling wave equations. The Fourier/Laplace transform is essentially a tool for time domain to frequency domain translation, and vice versa. In that transformation, the dependence of each component of the original signal on time is described by a timerelated constant: the angular frequency $\omega$. The transformation then maps the time domain into the $\omega$ domain, essentially by finding correlations between the original signal and an infinite set of pure sinusoidal signals. Those correlations collectively become a "frequencydomain" representation of the signal. In the class of signals represented by traveling-wave equations, however, the signal has existence in two domains: the time domain $t$ and the spatial domain $x$. More specifically, the signal has dependence on two propagation constants: the temporal propagation constant $\omega$, and the spatial propagation constant $k[1,2]$. Hence, a 
transformation is needed to map the behavior of the signal in the $x-t$ domain into the $k-\omega$ domain. The Fourier/Laplace transform only maps the $t$ domain into the $\omega$ domain and is incapable of performing the $2 \times 2$ mapping that is required in this case. Here, it is important to point out that the transform known as the "multidimensional Fourier transform" [3], used extensively in image processing applications, is still a $1 \times 1$ mapping process. By its definition, the "multidimensional Fourier transform" cannot perform a transformation of a signal that has simultaneous existence in both the time and the spatial domains.

The first objective of this paper is to present an important extension to the Fourier/Laplace transform that will allow the $2 \times 2$ mapping that is required in the case of traveling-wave signals to be performed. Another objective of the paper is to demonstrate that certain partial fraction decomposition and differentiation techniques can obviate the need for complex computations to simulate the behavior of large systems of optical oscillators. More particularly, some optical phenomena, such as the phenomenon of optical echo, are very hard to simulate without using physical variables corresponding to the internal states of a great number of atoms. Typically, such simulations require complex (order $N^{2}$, where $N$ is the number of atoms) computations. We demonstrate that this problem can be fundamentally solved in a much simpler manner by using derivative-taking procedures for symbolic polynomials.

\section{A Dual-Domain Extension for the Fourier/Laplace Transform}

Signals that physically propagate in various media are usually represented by traveling-wave equations, the most basic of which is the simple sinusoidal traveling wave $[1,2]$ :

$$
f(x, t)=\sin (k x-\omega t)
$$

where $\omega$ is the usual temporal propagation constant and where $k=2 \pi / \lambda$ is the spatial propagation constant. The usual Fourier transform for a signal that exists only in the time domain is given by $[4,5]$

$$
f(\omega)=\int_{-\infty}^{\infty} f(t) e^{-j \omega t} d t
$$

We first define a companion to the Fourier transform for signals that are defined only in the spatial domain:

$$
f(k)=\int_{-\infty}^{\infty} f(x) e^{+j k x} d x
$$

This transform maps the $x$ domain into the propagation constant $(k)$ domain. We now introduce a $2 \times 2$ dual-domain transform as follows:

$$
f(k, \omega)=\iint_{-\infty}^{\infty} f(x, t) e^{j(k x-\omega t)} d x d t .
$$


Notice that the Fourier transform measures the correlations between the signal $f(t)$ and an infinite set of pure sinusoidal signals in the time domain, while the transform of (2.4) measures the correlations between the signal $f(x, t)$ and an infinite set of pure sinusoidal traveling waves. This is the main difference between the transform of (2.4) and the other known extensions to the Fourier transform.

To write the one-sided version of the transform in (2.4) (which will be equivalent to the Laplace transform), we first decompose the exponential term as follows:

$$
e^{j(k x-\omega t)}=e^{\gamma x} e^{-s t}
$$

where $r=j k$ and $s=j \omega$. The one-sided transform will now be written as follows:

$$
f(k, \omega)=\iint_{0}^{\infty} f(x, t) e^{\gamma x} e^{-s t} d x d t
$$

We can now clearly see that this dual-domain transform differs from the basic Laplace transform in that an extra exponential term $e^{r x}$ is introduced, with $\gamma$ being an imaginary number representation of the spatial propagation constant $k$. The basic Laplace transform contains the imaginary number $s$ only, which is a representation of the temporal propagation constant $\omega$.

\section{Dual-Domain Transforms of Some Well-Known Propagating Signals}

We will now demonstrate how the dual-domain transform is calculated for various propagating signals. The transforms of two well-known signals, specifically, the propagating sinusoid and the propagating unit-step function are calculated here as examples.

\subsection{Transform of $f(x, t)=\sin (a x-b t)$}

By using the well-known decomposition

$$
\sin \theta=\frac{e^{j \theta}-e^{-j \theta}}{2 j}
$$

and carrying out the integration in (2.6) in a straightforward manner, the dual-domain transform of $f(x, t)=\sin (a x-b t)$ is found to be the following:

$$
f(k, \omega)=\frac{a s+b r}{\left(s^{2}+b^{2}\right)\left(\gamma^{2}+a^{2}\right)} .
$$

We can clearly see that if $a=0$, this transform reverts to the Laplace transform, with the presence of $\gamma=j k$ in the denominator. Essentially, what we get in that case will be the Laplace transform of $\sin b t$, mapped into the $k$ domain. 


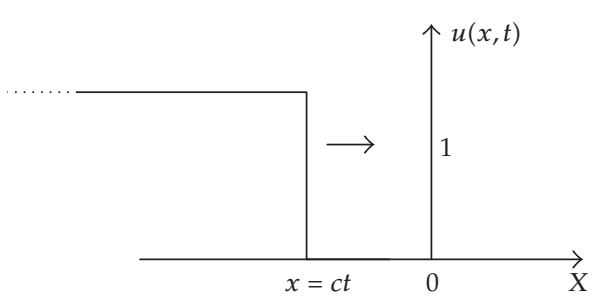

Figure 1: A propagating unit step function.

\subsection{Transform of a Propagating Unit Step Function $f(x, t)=u(x-c t)$}

A propagating unit step function $u(x-c t)$ is shown in Figure 1 . The velocity of propagation of the wave is $c$.

Here, due to the positive exponent of $e^{\gamma x}$ in (2.6), we will reverse the order of the limits on the integrals in order to obtain a meaningful result. Specifically, the time domain will be assumed to extend from $-\infty$ to 0 and the $x$ domain will be assumed to extend from $-\infty$ to $c t$. The transform of (2.6) will now be written as

$$
f(k, \omega)=\int_{t=-\infty}^{t=0} \int_{x=-\infty}^{x=c t} 1 \cdot e^{\gamma x} e^{-s t} d x d t
$$

The result is

$$
f(k, \omega)=\frac{1}{r(r c-s)} .
$$

We can again easily observe that if the propagation velocity $c=0$, the Laplace transform of a stationary unit step function is obtained, with the presence of an extra factor of $1 / \gamma$ that represents the mapping into the $k$ domain.

\section{Dual-Domain Transform Theorems}

We will now present two important theorems related to the dual-domain transform. Those theorems, namely, the phase-shift theorem and the convolution theorem, are straightforward extensions to the corresponding theorems of the Fourier/Laplace transforms.

\subsection{The Phase-Shift Theorem}

The dual-domain transform of a signal that is phase shifted by a phase $\phi=k \Delta x-\omega \Delta t$, where $\Delta x$ and $\Delta t$ are shifts along the $x$ and $t$ dimensions, respectively, is given by

$$
D[f(x-\Delta x, t-\Delta t)]=e^{j \phi} f(k, \omega),
$$

where $f(k, \omega)=D[f(x, t)]$ is the dual-domain transform of $f(x, t)$. 
Proof. One has

$$
D[f(x-\Delta x, t-\Delta t)]=\iint_{0}^{\infty} f(x-\Delta x, t-\Delta t) e^{\gamma x} e^{-s t} d x d t
$$

Let $(t-\Delta t)=\tau$ and $(x-\Delta x)=\delta$, so that $d t=d \tau$ and $d x=d \delta$. Hence

$$
\begin{aligned}
D[f(x-\Delta x, t-\Delta t)] & =\iint_{0}^{\infty} f(\delta, \tau) e^{\gamma(\delta+\Delta x)} e^{-s(\tau+\Delta t)} d \tau d \delta \\
& =e^{(\gamma \Delta x-s \Delta t)} \iint_{0}^{\infty} f(\delta, \tau) e^{\gamma \delta} e^{-s \tau} d \tau d \delta \\
& =e^{j(k \Delta x-\omega \Delta t)} f(k, \omega) .
\end{aligned}
$$

\subsection{The Convolution Theorem}

A signal $z(x, t)$, the dual-domain transform of which is given by $z(k, \omega)=y(k, \omega) h(k, \omega)$, where $y(k, \omega)$ and $h(k, \omega)$ are the transforms of another signal and a transfer function, respectively, is given by

$$
z(x, t)=\iint_{-\infty}^{\infty} y(\delta, \tau) h(x-\delta, t-\tau) d \delta d \tau
$$

Proof. $z(x, t)$ will be given by the inverse dual-domain transform of $y(k, \omega) h(k, \omega)$, that is,

$$
\begin{aligned}
z(x, t) & =D^{-1}[y(k, \omega) h(k, \omega)] \\
& =\iint_{-\infty}^{\infty} y(k, \omega) h(k, \omega) e^{-j(k x-\omega t)} d k d \omega .
\end{aligned}
$$

But

$$
y(k, \omega)=\iint_{-\infty}^{\infty} y(\delta, \tau) e^{j(k \delta-\omega \tau)} d \delta d \tau
$$

Hence

$$
z(x, t)=\iint_{-\infty}^{\infty} \iint_{-\infty}^{\infty} y(\delta, \tau) e^{j(k \delta-\omega \tau)} d \delta d \tau h(k, \omega) e^{-j(k x-\omega t)} d k d \omega .
$$


By interchanging the order of the double integrals, we have

$$
\begin{aligned}
z(x, t) & =\iint_{-\infty}^{\infty} y(\delta, \tau)\left[\iint_{-\infty}^{\infty} h(k, \omega) e^{-j[k(x-\delta)-\omega(t-\tau)]} d k d \omega\right] d \delta d \tau \\
& =\iint_{-\infty}^{\infty} y(\delta, \tau) h(x-\delta, t-\tau) d \delta d \tau .
\end{aligned}
$$

\section{Example Application: Transfer Function of a Wireless Communication Channel with Multiple Phase Shifts}

The transfer function characteristics of urban wireless communications channels are very important for cellular phone and wireless multimedia applications [6-8]. The high-frequency signals that travel through such channels are all represented by traveling wave equations, and each signal suffers from various phase shifts that collectively result in "fading effects" as the signal travels through the channel. Characterizing the behavior of a channel by means of a transfer function has always been a challenging problem, mainly because the Fourier/Laplace transform can only provide a transfer function representation in the frequency domain, that is, the $\omega$ domain, while the behavior of the channel in reality must ultimately be described in both the $\omega$ and the $k$ domains. We will now proceed to obtain such a dual-domain transfer function for a wireless communication channel by using the dualdomain transform, and important new conclusions about the behavior of such channels will be reached.

According to the phase-shift theorem (4.1), a signal that encounters multiple phase shifts in a wireless communication channel will be represented by the sum

$$
A_{0} e^{j \phi_{0}} f(k, \omega)+A_{1} e^{j \phi_{1}} f(k, \omega)+A_{2} e^{j \phi_{2}} f(k, \omega)+\cdots
$$

at the output of the channel, where $\phi_{i}$ are the various phase shifts and where $A_{i}$ are the amplitudes of the phase-shifted components. The transfer function (TF) of the channel is therefore given by

$$
\mathrm{TF}=\frac{\text { Channel Output }}{f(k, \omega)}=A_{0} e^{j \phi_{0}}+A_{1} e^{j \phi_{1}}+A_{2} e^{j \phi_{2}}+\cdots
$$

By expanding each exponential term as a power series and rearranging the equation, we get

$$
\mathrm{TF}=\left(A_{0}+A_{1}+\cdots\right)+j\left(A_{0} \phi_{0}+A_{1} \phi_{1}+\cdots\right)+\frac{j^{2}}{2 !}\left(A_{0} \phi_{0}^{2}+A_{1} \phi_{1}^{2}+\cdots\right)+\cdots
$$

For comparison, the single-domain Laplace transform of a rectangular pulse in the time domain is given by [5]

$$
f(\omega)=C_{0}-C_{1} s+C_{2} s^{2}-\cdots,
$$




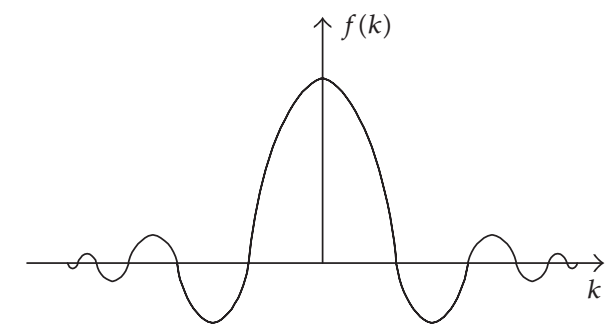

Figure 2: Behavior of the multipath wireless channel in the $k$ domain.

where $C_{i}$ are constants. It is clear that while (5.4) is a power series in $j \omega,(5.3)$ is a power series in $j \phi$. This means that (5.3) is the transform of pulse-like characteristics in the time domain and pulse-like characteristics in the spatial domain. This is a very important conclusion, since it was previously assumed that multipath wireless channels exhibit pulse-like characteristics in the time domain only [6]. The pulse-like behavior of the channel in the spatial domain will be then described by a sinc function in the $k$ domain, as shown in Figure 2.

The conclusion therefore is that the channel acts as a band-pass filter in both the frequency domain and the propagation constant $k$ domain. This conclusion has substantial implications for the analysis of multipath wireless channels and will be further developed and investigated in a subsequent report in a specialized communications journal.

\section{Aspects Regarding the Propagation of Traveling Waves Corresponding to Wave Functions. Phase Loss due to Multiple Quantum Interaction}

We can extend our study at quantum phenomena by taking into account the fact that the expression $(k x-\omega t)$ corresponds also to the phase of a wave function describing the propagation of a free quantum particle.

According to the statistical interpretation of quantum theory, the wave function is representing just a mathematical model suitable for statistical interpretation. In the David Bohm interpretation of quantum theory, the wave function is a real wave in configuration space. This interpretation is also in accordance with measurements aspects of interaction of wave-trains with observer's material medium [9]. Due to these reasons, the phase of the traveling quantum wave requires a more detailed analysis, the dynamical aspect having to be taken into consideration.

Let us consider the propagation of an electron in a central symmetric electrostatic (Coulomb) field. According to the standard quantum analysis (based on second quantification theory, describing creation and annihilation of particles) the central symmetric electrostatic field should be decomposed (using the Fourier transformation) in a set of waves with a certain angular frequency $\omega$ and a certain wave vector $k$ :

$$
A_{\mu}^{e}\left(x_{0}, \vec{x}\right)=\frac{1}{(2 \pi)^{2}} \int d q A_{\mu}^{e}(q) \exp -i q x
$$


where the product $q x$ stands for

$$
q x=q_{0} x_{0}-\vec{q} \vec{x}
$$

with $x_{0}$ representing the time coordinate, $\vec{x}=x_{1}, x_{2}, x_{3}$ representing to the vector of position, $q_{0}$ representing the angular frequency , and $\vec{q}=q_{1}, q_{2}, q_{3}$ representing the wave vector. The measurement system is chosen so as $c=1, h /(2 \pi)=1$ for performing a better correspondence from the angular frequency to the energy and from the wave vector to the momentum of the quantum particle (see [10] for more details).

The quantities $A_{\mu}^{e}(x)$ and $A_{\mu}^{e}(p)$ do not correspond to standard photons, and for this reason they cannot be substituted by operators as required by second quantification theory. However, experimental facts have shown that the electromagnetic field effect can be studied using the perturbations method from quantum theory.

Using this method [10] the matrix element (for the first-order of perturbation) corresponding to the electromagnetic interaction between the electron and the electromagnetic field is represented by,

$$
S_{f i}^{(1)}=-i e \int d x \bar{v}_{r^{\prime}}^{(+)}\left(\vec{p}_{f}\right) \exp i p_{f} x \int d q A_{\mu}^{e}(q) \gamma_{\mu} \exp -i q x v_{r}^{(-)}\left(\vec{p}_{i}\right) \exp -i p_{i} x,
$$

which can be also written as,

$$
S_{f i}^{(1)}=-i e \int d q \bar{v}_{r^{\prime}}^{(+)}\left(\vec{p}_{f}\right) \gamma_{\mu} A_{\mu}^{e}(q) v_{r}^{(-)}\left(\vec{p}_{i}\right) \delta\left(q-p_{f}+p_{i}\right)
$$

In both previous equations the expression $A_{\mu}^{e} \gamma_{\mu}$ corresponds to the sum:

$$
A_{\mu}^{e} \gamma_{\mu}=A_{0}^{e} \gamma_{0}+\sum_{n=1}^{3} A_{n}^{e} \gamma_{n}
$$

where $A_{\mu}^{e}$ represents the cuadrivector of the electromagnetic field and $\gamma_{\mu}$ represents the Dirac matrices (its argument corresponds to the energy and momentum conservation laws).

If the electromagnetic field does not depend on time, the cuadripotential $A_{\mu}^{e}(x)$ can be presented as

$$
A_{\mu}^{e}(x)=A_{\mu}^{e}(\vec{x})=\frac{1}{(2 \pi)^{3 / 2}} \int d q \exp i \vec{q} \vec{x} A_{\mu}^{e}(\vec{q})
$$

which shows that the virtual photons composing the electromagnetic field have a nonzero value just for the momentum (the energy corresponding to quantity $q_{0}$ being equal to zero). As a consequence, the matrix element for the first-order of perturbation can be written as

$$
S_{f i}^{(1)}=-i e \int d q \bar{v}_{r^{\prime}}^{(+)}\left(\vec{p}_{f}\right) \gamma_{\mu} A_{\mu}^{e}(\vec{q}) v_{r}^{(-)}\left(\vec{p}_{i}\right) \delta\left(\vec{q}-\vec{p}_{f}+\vec{p}_{i}\right) \delta\left(p_{f 0}-p_{i 0}\right)
$$


By performing the integration on $d q$, it results

$$
S_{f i}^{(1)}=-i e \bar{v}_{r^{\prime}}^{(+)}\left(\vec{p}_{f}\right) \gamma_{\mu} A_{\mu}^{e}(\vec{q}) v_{r}^{(-)}\left(\vec{p}_{i}\right) \delta\left(p_{f 0}-p_{i 0}\right)
$$

For the coulombian electrostatic field with central symmetry $A_{\mu}^{e}(\vec{x})=0$; it results

$$
e A_{0}^{e}(r)=-\frac{Q}{r}
$$

where $Q$ represents the electrical negative charge. The previous equation can be also written (in $p$ representation) as

$$
e A_{0}^{e}(r)=-\int \frac{Q}{r} \exp i q r d r=\frac{4 \pi Q}{\vec{q}^{2}} .
$$

As a consequence, the matrix element for the first-order of perturbation in case of coulombian field with central symmetry can be written as:

$$
S_{f i}^{(1)}=-i e \bar{v}_{r^{\prime}}^{(+)}\left(\vec{p}_{f}\right) \gamma_{0} A_{0}^{e}(\vec{q}) v_{r}^{(-)}\left(\vec{p}_{i}\right) \delta\left(p_{f 0}-p_{i 0}\right),
$$

which can be also written as

$$
S_{f i}^{(1)}=-i \bar{v}_{r^{\prime}}^{(+)}\left(\vec{p}_{f}\right) \gamma_{0} v_{r}^{(-)}\left(\vec{p}_{i}\right)\left[e A_{0}^{e}(\vec{q})\right] \delta\left(p_{f 0}-p_{i 0}\right)
$$

According to standard interpretation of quantum theory, this first-order element from perturbation method is connected to the probability of an interaction between an electron with initial momentum $\vec{p}_{i}$ and energy $p_{i 0}$ and a virtual photon with momentum $\vec{q}$ so as to result an electron with momentum $\vec{p}_{f}$ and energy $p_{f 0}$. This interaction can be also represented using Feynman diagrams. By analyzing higher orders of matrix element (presented in an intutive manner using the same Feynman diagrams) some supplementary phenomena can be also explained, as elastic diffusion of photons by electrons, the annihilation of an electron-positron pair in two photons, the generation of an electron-positron pair by photons, the emission of two photons by an electron, the emission of brehmstrahlung radiation by electrons and positrons and so on.

However, for describing the whole interaction of an electron in a central symmetrical electrostatic field (so as to determine the trajectory of the associated wave-train) we can consider that the electron has to undergo multiple interactions with such virtual photons until the action of the exterior field vanishes (such multiple interactions being allowed by quantum mechanics). According to the quantum laws, each interaction transform the initial electron (corresponding to a certain wave train) into a new wave-train (the final electron) with different characteristics (another momentum and energy), and so on.

However, we should take also into account the fact that the wave-train associated to a quantum particle has not just a statistical interpretation. The Bohm-Aharonov effect has shown that a certain phase should also be associated to such a wave-train. Considering 
that the wave-train corresponding to an electron undergoes a set of interactions with virtual photons, it results that a certain transient time is required by each interaction, so as physical quantities as wavelength and angular fequency to be defined on a certain time interval (these quantities being used by the formula of matrix elements of interaction). This transient time causes a phase-loss for the initial wave-train, while the timelength of stabilized oscillations (corresponding to the new-generated wave-train associated to the final photon) is represented (in a classical dynamical model) by the difference between the timelength of the initial wave-train and the transient time. Considering that no supplementary stabilized oscillations are added by each interaction (this means that quantum interaction does not generate any output wave-train after the input wave-train has vanished) it results that a very great number of interactions of the initial electron in a central symmetrical electrostatic (coulombian) field would cause a great number of phase loss (each interaction substracting a certain transient time), and, finally, the timelength of the wave-train associated to the final electron tends to zero. This corresponds to a vanishing phenomena for the initial electron in contradiction with experimental facts.

\section{Nonlinear Phenomena of Phase-Loss Compensation for Propagating Wave-Trains in Quantum Physics: The Need of a Certain Material Reference System}

At an extended analysis of propagating phenomena in quantum physics, we can notice that an approach based on second quantification laws leads to the same aspects of phase-loss (or shortened timelength) of a propagating wave-train due to multiple interactions along its trajectory in different situations, such as the interaction of a propagating free electron with its own electromagnetic field (implying renormalization of electron mass using higher orders of perturbation theory) and the generation of virtual electron-positron pairs due to the interaction of an electron with a weak exterior electromagnetic field (analyzed by taking into account higher order of perturbation theory, implying renormalization of electron charge) [10].

For justifying the fact that the phase-loss does not appear in experimental phenomena of such propagating beams (as presented above), we can take into consideration a possible phenomenon of phase-addition, consisting in an addition of supplementary oscillations after the initial wave-train has vanished. According to mathematical dynamical models, this corresponds to a dead-time transient phenomenon. As a consequence, a kind of internal memory should be associated to the space interval where the interaction occurs, generating an output signal even after the input signal has disappeared.

Another possibility consists in taking into account (from the same dynamical point of view) a resonance phenomena, considering that the initial wave-train can generate (as in a resonant phenomenon) oscillations with amplitude above a certain threshold. When the initial wave-train (similar to an input signal) disappears, these oscillations will become damped oscillations able to generate a final wave-train (similar to an output signal) as long as their amplitude is above a certain threshold. As in the case of the previous dynamical model, this mathematical model requires also an internal memory of the threshold level and of certain state variables able to generate resonant oscillations.

Both possibilities previously presented have a common feature: a certain delay time appears at each interaction, and thus the overall delay time should be connected with the wave-train velocity. 
However, the phase-loss phenomenon can be avoided (as a theoretical result) if the whole trajectory is considered to be determined by just one Lagrangean function for the whole interaction of the electron with the electromagnetic field, implying the dynamical equations:

$$
\begin{gathered}
\left(-\frac{\partial^{2}}{\partial t^{2}}+\nabla^{2}\right) A_{\mu}(x)=-e \bar{\psi} \gamma_{\mu} \psi, \\
\left(i \gamma_{\mu} \frac{\partial}{\partial x_{\mu}}-e \gamma_{\mu} A_{\mu} \psi(x)\right)-m \psi(x)=0 \\
\bar{\psi}(x)\left(i \gamma_{\mu} \frac{\partial}{\partial x_{\mu}}+e \gamma_{\mu} A_{\mu}\right)+m \bar{\psi}(x)=0
\end{gathered}
$$

[10]. Thus the wave-function corresponding to the electron and the electromagnetic field is modified in a continuous manner along its trajectory. However, this possible solution is based on quantum laws of first quantification theory, all phenomena corresponding to second quantification (involving creation and annihilation of particles) being neglected. Yet we have to take into account the fact that the exterior electromagnetic field has usually a higher magnitude and a slow time variation as compared to the energy and velocity of the interacting electron. So it is quite naturally to consider that a certain exterior field (more important at a macrosopic scale) is determining the evolution of the electron wavetrain, and thus appears the need of a certain reference system which acts upon the wavetrains corresponding to quantum particles. This assumption is supported also by the lack of reversibility for diffraction phenomena in quantum physics. We can notice an electron diffraction phenomenon when an electron beam interacts with a motionless crystal lattice, but we cannot imagine a diffraction phenomenon for the atoms of a crystal lattice when a moving lattice interacts with a motionless spatial distribution of electrons.

\section{Phase Aspects and Quantum Dynamics of Cooperative Phenomena: Formal Logic Problems for Cooperative Quantum Phenomena}

Our study upon quantum dynamics and phase aspects should be extended by taking into consideration multiparticle cooperative phenomena in quantum physics involving emission of electromagnetic field, while key issues as transition from a set of single particle quantum states to a global cooperative state can be noticed. For example, the photonic echo phenomenon is based on the rephasing that can occur in an inhomogeneously broadened atomic system, when the motion of the Bloch vectors is reversed following the initial dephasing of the atomic dipoles. For the beginning, we consider a group of atoms whose Bloch vectors in the rotating frame have the direction along the $y^{\prime}$ axis at the zero moment of time. We consider also that the rotating frame has the angular frequency $\omega_{0}$ (the central frequency of the inhomogeneous line). In time, the Bloch vectors of the atoms will precess clockwise or counter-clockwise around the $z^{\prime}$ axis, depending whether their natural angular frequencies are either greater or less than $\omega_{0}$. In time a dephasing phenomenon occurs, the atomic Bloch vectors being distributed almost uniformly around the $x^{\prime}, y^{\prime}$ plane. The initial macroscopic moment becomes zero, and the system does not radiate. 
By applying a short, intense $\pi$ pulse at time moment $\tau$ a reversal of the $y^{\prime}$ coordinate appears, the motion of the atomic Bloch vectors is reversed, and the vectors would be aligned again at a certain time moment $2 \tau$. As a consequence, the atomic system generates a high-intensity echo pulse at time moment $2 \tau$. This is the basic principle of both spin echo phenomenon and photon echo phenomenon [11].

Usually, a preparatory $\pi / 2$ pulse (from the superradiance states point of view) is applied to the active medium at the initial moment of time. Mathematically, the property of the reference pulse of being a $\pi / 2$ pulse is represented by the relation

$$
\eta E_{I} \Delta t_{I}=\frac{\pi}{2}
$$

where $E_{I}$ stands for the amplitude of the electric field, $\Delta t_{I}$ stands for its timelength and $\eta$ represents a parameter depending on frequency. The condition for the main optical pulse of being a $\pi$ pulse is represented by the relation

$$
\eta E_{M} \Delta t_{M}=\pi
$$

where $E_{M}$ stands for the electric field of this signal, $\Delta t_{M}$ stands for its timelength, and $\eta$ represents the same parameter depending on frequency.

A basic feature of the high-intensity pulse (the photonic echo pulse) is represented by its power. Unlike amplified optical pulses by stimulated emission (with the power of emergent pulse proportional to $N$ ), the power of the photonic echo pulse is proportional to $N^{2}$ where $N$ represents the number of atoms involved in the emission of this high-intensity pulse. This feature can be derived using the formalism of superradiant states $|r, m\rangle$, where $r$ stands for the cooperative quantum number and $m$ is proportional to the unperturbed Hamiltonian function for the ensemble of atoms (due to the the similarities with quantic angular momentum theory, $|m|<r<N / 2$ ). According to this similarities, when the state of the atomic system is described by the state $|r, m\rangle$, the intensity of the emitted pulse is

$$
I(r, m)=(r+m)(r-m+1) I_{0} .
$$

If all atoms involved in light emission are in excited state, $r=m=N / 2$ and the intensity of emitted pulse is equal to

$$
I\left(\frac{N}{2}, \frac{N}{2}\right)=N I_{0}
$$

where $I_{0}$ stands for the intensity of an optical pulse emitted by a single atom $(r=m=1 / 2)$. It can be shown that the maximum for the emission rate is obtained for $m=1 / 2$ and greater values of $r$; while $m$ is also equal to $\left(N_{+}-N_{-}\right)\left(N_{+}\right.$representing the number of atoms in excited state and $N_{-}$representing the number of atoms in ground state) it results that $m=0$ 
or $m=1$ and $r=N / 2$. In this case the intensity of emitted pulse is

$$
I\left(\frac{N}{2}, 0\right)=I\left(\frac{N}{2}, 1\right)=\left(\frac{N}{2}\right)\left(\frac{N}{2}+1\right) I_{0}
$$

being proportional (for large $N$ ) with $N^{2}$.

These aspects can be also explained (in an intuitive manner) using classical electromagnetics. When all atoms radiate independently, it can be considered that the phase $\phi$ and angular frequency $\omega$ of emitted electric field are not the same and thus the average value of the intensity corresponds to a sum of $a_{k}^{2} E_{k}^{2} / 2$ terms (all terms having the form $a_{i} a_{k} E_{i} E_{k} / 2$ vanish while they correspond to alternating functions). It can be also argued that different orientation of Bloch vectors for a great number of atoms imply a null result for the sum of these vectors from the very beginning. On the contrary, when all atoms radiate in a cooperative manner, it can be considered that their emitted electric field has the same phase and angular frequency and thus the electric field $E$ generated by all atoms is represented by a sum $E_{1}+E_{2}+\cdots+E_{N}$ and the intensity of the emitted electric field is represented by $\left(E_{1}+E_{2}+\cdots+E_{N}\right)^{2}$. For $E_{1}=E_{2}=\cdots=E_{N}$ it results that the intensity of the emitted electric field is proportional to $N$ (in the first case) and to $N^{2}$ (in the second case). It can be also argued that the situation when all Bloch vectors are aligned corresponds to a great global dipole momentum for the entire system of atoms, and thus the emitted radiation has the same phase.

However, an important question regarding phase appears for these cases: which is the angular frequency of the emitted field for the photonic echo phenomenon? Is there a certain angular frequency or should we consider the emitted field as a mixture of waves with different characteristics? If we consider that we deal with a cooperative phenomenon, it seems logical to search for a certain angular frequency and a certain phase for the whole emitted field (as in the case of correlated photon emission, when two photons act as a single entity even when they are situated at large distances). Thus the emission would be similar to a single photon emission and interference phenomena could be noticed further (according to the Dirac principle that a photon interacts just with itself). Yet this affirmative answer generates another question: under which circumstances an ensemble of atoms can be studied using the quantum formalism of superradiant states, instead of analyzing a superposition of atoms with independent emission? According to formal logic in physics, an interference phenomenon can either occur or not. However, it is quite difficult to imagine that a certain parameter of the ensemble could suddenly generate the transition to a single quantum state when it reaches a specific value (so as interference phenomena to occur). This aspect is similar to the problem of defining a certain material reference system which performs the Lorentz transformation upon a received wave-train [9]: which are the requirements for a certain material reference system (as related to the parameters of the received wavetrain)? (see [12] for the theoretical case when the energy of the received wave is comparable to the rest energy of the atoms composing the material medium). More probably, some correlation techniques with past events are required, and thus phase correlation aspects could appear. The phase correlation represents also the main technique for creating and controlling cooperative phenomena (when some spatially distributed particles or atoms can be described by a single quantum function at a certain moment of time, and possible transitions are controlled by imposing phase coincidence). 


\section{Simulating High-Intensity Superradiant Pulses Using Derivative Procedures and Partial Fraction Decomposition}

A step forward in looking for an adequate mathematical model should consist in finding a mathematical quantity proportional to $(N / 2)(N / 2-1)$; this step must be performed in a similar manner used for passing from coordinates representation to momentum representation in quantum theory, by the aid of Fourrier transformation (the basic variables being changed) [13]. We can easily notice that the derivatives of different order for $f=p^{N / 2}$ are direct proportional to $N / 2,(N / 2)(N / 2-1), \ldots$ according to

$$
\begin{gathered}
\frac{d f}{d p}=\left(\frac{N}{2}\right) p^{N / 2-1}, \\
\frac{d^{2} f}{d p^{2}}=\left(\frac{N}{2}\right)\left(\frac{N}{2}-1\right) p^{N / 2-2} .
\end{gathered}
$$

It can be noticed that the coefficient of final monomial $p^{N / 2-2}$ equals $(N / 2)(N / 2-1)$ which represents the ratio of the intensity $I(N / 2, N / 2)$ of the photonic echo pulse emitted from the state $r=m=N / 2$ to the intensity $I_{0}$ of the pulse emitted independently by a single atom (similar to derivative procedures presented in [14]). In an intuitive manner, $p$ can be considered as an operator of creation (as in second quantification formula) and the $N / 2$ exponent symbolizes the creation of the quantum superradiant state with quantum number $r=N / 2$ as a result of $N / 2$ successive application of $p$ operator upon an initial preliminary state (with $r=0$ ). The same derivative procedure could be used for obtaining the eigenvalue of the square of the angular momentum $\widehat{L}^{2}=L(L+1)$ according to

$$
\left(\frac{\partial^{2}}{\partial p^{2}}\right) p^{L+1}=(L+1) L p^{L-2}
$$

and considering an eigenvalue the coefficient of the resulting monomial.

For the case of nonsuperradiant states the ensemble of atoms could be described using a function $f$ as

$$
f=\sum_{i}\left(p-\varphi_{i}\right)
$$

where $\varphi_{i}$ stands for the specific phase difference for each atom. While the power of $p$ equals unity, a second-order differentiation $\partial^{2} f / \partial p^{2}$ yields zero, and no superradiant pulse appears.

\section{Conclusions}

This paper has presented an important extension to the Fourier/Laplace transform that is needed for the analysis of signals that are represented by traveling wave equations. Specific mathematical technique for the simulation of the behavior of large systems of optical oscillators was obtained. Signals described by traveling wave equations are typically characterized by a spatial propagation constant, $k$, in addition to the usual temporal 
propagation constant $\omega$. The Fourier/Laplace transform can only perform mapping of the time domain $t$ into the $\omega$ domain (or frequency domain), and is incapable of performing the more general $2 \times 2$ mapping of the $x-t$ domain into the $k-\omega$ domain. The new extension introduced here allows for that general $2 \times 2$ mapping to be performed. This study has also emphasized the importance of phase dynamics aspects for traveling wave-trains in multiple interaction phenomena in quantum physics. It was shown that a phase-loss phenomenon should appear due to transient time, implying an annihilation of the quantum particle due to multiple interactions. The absence of this phenomenon in experimental results is explained by a possible correlation between the trajectory and phase of the quantum wave-train before interaction and the trajectory and phase of the quantum wave-train after the corresponding quantum particle has undergone the interaction. The need of a certain exterior reference system acting upon the quantum wave-train is also presented (based on some diffraction aspects). Finally it is shown that some interference aspects of the photonic echo phenomenon imply the need of considering that superradiant quantum states for an ensemble of atoms can either exist or not. Using a specific differentiation technique for symbolic polynomials corresponding to phase difference of each atom, a useful formalism for determining the condition for generating a superradiant pulse and its intensity is obtained.

\section{References}

[1] W. H. Hayt and J. A. Buck, Engineering Electromagnetics, McGraw Hill, New York, NY, USA, 2006.

[2] R. Feynmam, R. Leighton, and M. Sands, The Feynman Lectures on Physics, vol. 2, Addison Wesley, Reading, Mass, USA, 1977.

[3] J. S. Lim, Two-Dimensional Signal and Image Processing, Prentice Hall, Upper Saddle River, NJ, USA, 1990.

[4] T. W. Korner, Fourier Analysis, Cambridge University Press, Cambridge, UK, 2nd edition, 1989.

[5] J. E. Marsden and M. J. Hoffman, Basic Complex Analysis, W. H. Freeman, New York, NY, USA, 2nd edition, 1987.

[6] T. S. Rappaport, Wireless Communications, Prentice Hall, Upper Saddle River, NJ, USA, 1996.

[7] V. K. Garg and J. E. Wilkes, Wireless and Personal Communications Systems, Prentice Hall, Upper Saddle River, NJ, USA, 1996.

[8] V. K. Garg, Wireless Network Evolution, Prentice Hall, Upper Saddle River, NJ, USA, 2002.

[9] E. Bakhoum and C. Toma, "Relativistic short range phenomena and space-time aspects of pulse measurements," Mathematical Problems in Engineering, vol. 2008, Article ID 410156, 20 pages, 2008.

[10] N. Nelipa, Physique des Particules Elementaires, MIR, Moscou, Russia, 1981.

[11] L. Mandel and E. Wolf, Optical Coherence and Quantum Optics, Cambridge University Press, Cambridge, UK, 1995.

[12] A. Toma, S. Pusca, and C. Morarescu, "Spatial aspects of interaction between high-energy pulses and waves considered as suddenly emerging phenomena," in Proceedings of the 1st International Conference on Computational Science and Its Applications (ICCSA '06), vol. 3980 of Lecture Notes Computer Science, pp. 839-846, Glasgow, UK, May 2006.

[13] P. Sterian and C. Toma, "Methods for presenting key concepts in physics for MS students by photonMD program," Bulgarian Journal of Physics, vol. 27, no. 4, pp. 27-30, 2000.

[14] Th. Toma, S. Pusca, and C. Morarescu, "Simulating superradiant laser pulses using partial fraction decomposition and derivative procedures," in Proceedings of the 1st International Conference on Computational Science and Its Applications (ICCSA '06), vol. 3980 of Lecture Notes Computer Science, pp. 779-784, Glasgow, UK, May 2006. 


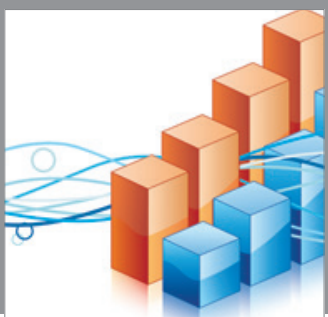

Advances in

Operations Research

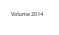

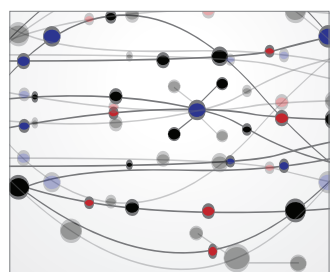

\section{The Scientific} World Journal
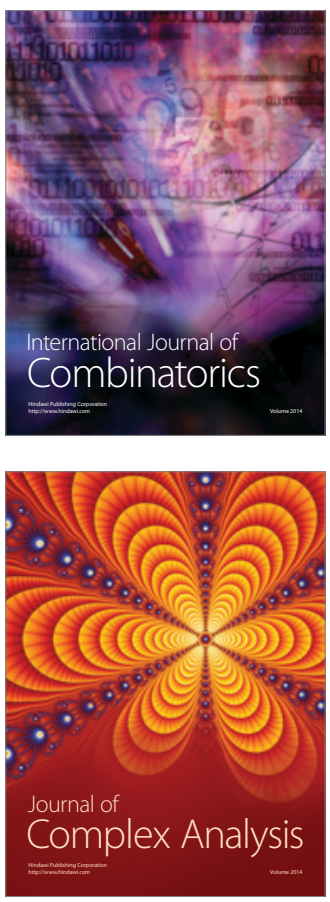

International Journal of

Mathematics and

Mathematical

Sciences
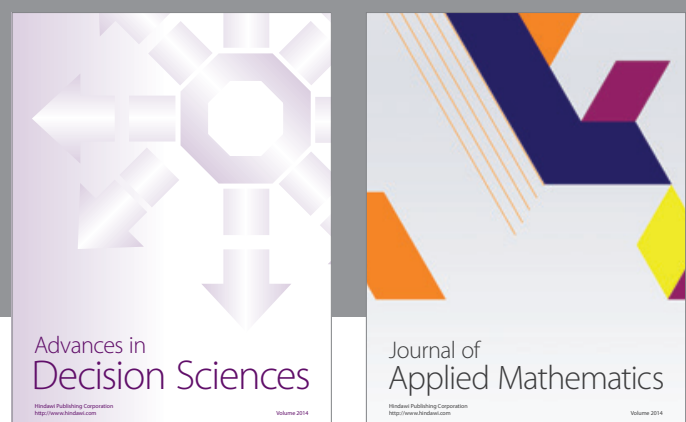

Journal of

Applied Mathematics
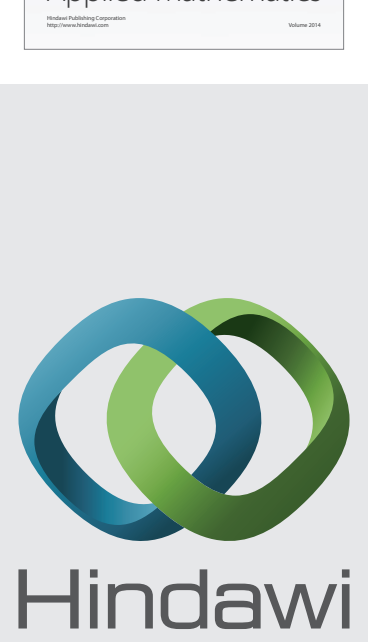

Submit your manuscripts at http://www.hindawi.com
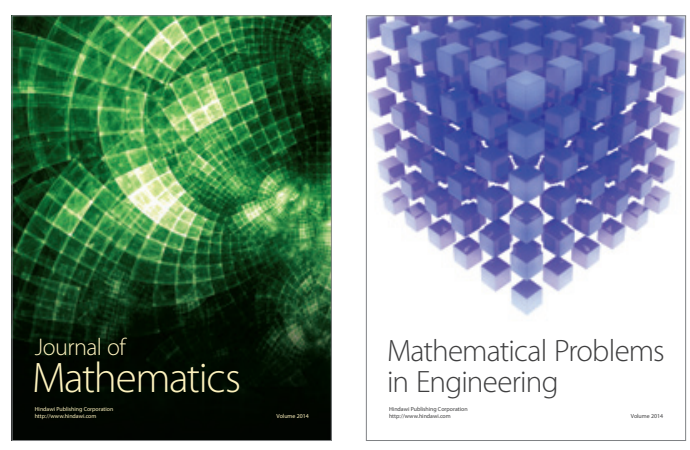

Mathematical Problems in Engineering
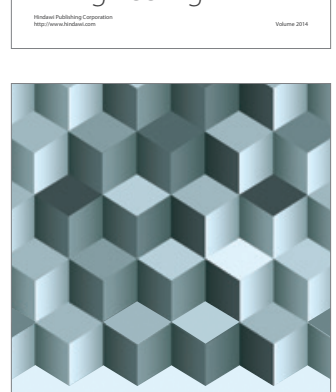

Journal of

Function Spaces
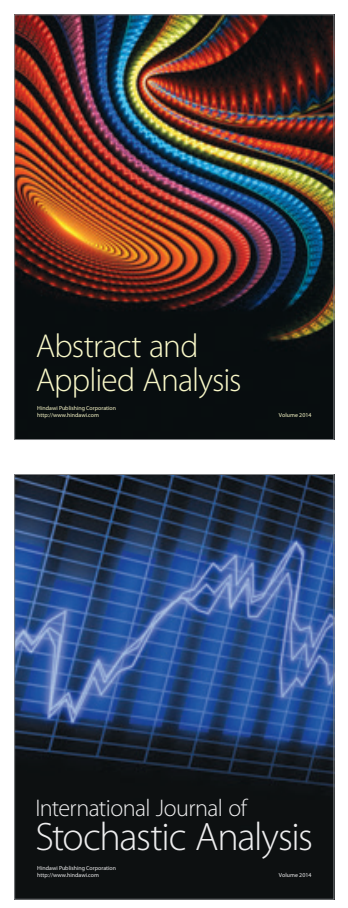

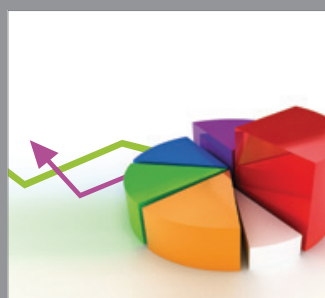

ournal of

Probability and Statistics

Promensencen
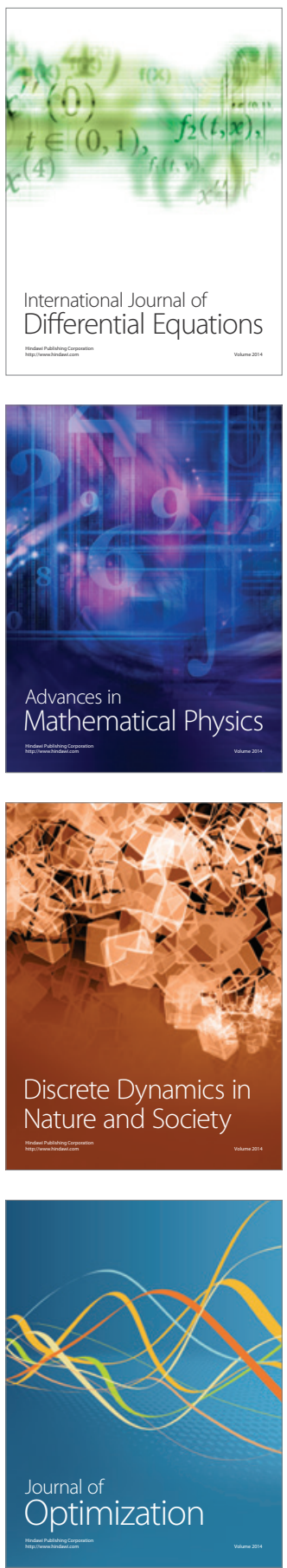